\title{
On the Laws of Total Local Times for $h$-Paths and Bridges of Symmetric Lévy Processes
}

\author{
Masafumi Hayashi' ${ }^{1}$ and Kouji Yano ${ }^{2}$ \\ ${ }^{1}$ Faculty of Science, University of the Ryukyus, 1 Senbaru, Okinawa, Nishihara 903-0213, Japan \\ ${ }^{2}$ Graduate School of Science, Kyoto University, Sakyo-ku, Kyoto 606-8502, Japan \\ Correspondence should be addressed to Kouji Yano; kyano@math.kyoto-u.ac.jp
}

Received 12 May 2012; Accepted 29 November 2012

Academic Editor: Dumitru Baleanu

Copyright (c) 2013 M. Hayashi and K. Yano. This is an open access article distributed under the Creative Commons Attribution License, which permits unrestricted use, distribution, and reproduction in any medium, provided the original work is properly cited.

The joint law of the total local times at two levels for $h$-paths of symmetric Lévy processes is shown to admit an explicit representation in terms of the laws of the squared Bessel processes of dimensions two and zero. The law of the total local time at a single level for bridges is also discussed.

\section{Introduction}

Markov processes associated to heat semigroups generated by fractional derivatives are called symmetric stable Lévy processes (cf., e.g., [1]) or Lévy flights (cf., e.g., [2]). The purpose of the present paper is to study the laws of the total local times for $h$-paths and bridges of (one-dimensional) symmetric Lévy processes. We give an explicit representation (Theorem 16) of the joint law as a weighted sum of the law of the squared Bessel process of dimension two and the generalized excursion measure for the squared Bessel process of dimension zero. We also give an expression (Theorem 20) of the law of the total local time at a single level for bridges.

It is well known as one of the Ray-Knight theorems (see, e.g., [3, Chapter XI] and [4, Chapter 3]) that the total local time process with space parameter for a Bessel process of dimension three is a squared Bessel process of dimension two. Since the Bessel process of dimension three is the $h$-path process of a reflected Brownian motion, Theorem 16 may be considered to be a slight generalization of this result.

Eisenbaum and Kaspi [5] have proved that the total local time of a Markov process with discontinuous paths is no longer Markov. As an analogue of Ray-Knight theorems, Eisenbaum et al. [6] have recently characterized the law of the local time process with space parameter at inverse local time in terms of some Gaussian process whose covariance is given by the resolvent density of the potential kernel. Moreover, if the Lévy process is a symmetric stable process, then the corresponding Gaussian process is a fractional Brownian motion. Their results are based on a version of Feynman-Kac formulae, which characterizes the Laplace transform of the joint laws of total local times of Markov processes at several levels.

In this paper we first focus on the $h$-path process of a symmetric Lévy process, which has been introduced in the recent works [7-9] by Yano et al. The $h$-path process may be obtained as the process conditioned to avoid the origin during the whole time (see [10]). We will also start from a version of Feynman-Kac formulae and obtain an explicit representation of the joint law of the total local times at two levels. (For some discussions of the joint law of the total local times, see Blumenthal-Getoor [11, pages 221-226] and Pitman [12].) Unfortunately, we have no better result on the law of the total local time process with space parameter. The difficulty will be explained in Remark 3.

In comparison with the results by Pitman [13] and Pitman and Yor [14] about the Brownian and Bessel bridges, we also investigate the law of the total local time at a single point for bridges of symmetric Lévy process, which we call Lévy bridges in short, and also for bridges of the $h$-paths, which we call $h$-bridges in short. We will prove a version of FeynmanKac formulae (Theorem 7) for Lévy bridges with the help of the general theorems by Fitzsimmons et al. [15]. As an application of the Feynman-Kac theorem, we will give an 
expression of the law of the total local time at a single level for the Lévy bridges, while, unfortunately, we do not have any nice formula for the $h$-bridges.

The present paper is organized as follows. In Section 2, we give two versions of Feynman-Kac formulae in general settings. In Section 3, we recall several formulae about squared Bessel processes and generalized excursion measures. In Section 4, we recall several facts about symmetric Lévy processes. In Section 5, we deal with the joint law of the total local times at two levels for the $h$-paths of symmetric Lévy processes. In Section 6, we study the laws of the total local times for the Lévy bridges and for the $h$-bridges.

\section{Feynman-Kac Formulae}

In order to study the laws of total local times, we prepare two versions of Feynman-Kac formulae, which describe their Laplace transforms. One is for transient Markov processes, and the other is for Markovian bridges.

Let $\mathbb{D}$ denote the space of càdlàg paths $\omega:[0, \infty) \rightarrow$ $\mathbb{R} \cup\{\Delta\}$ with lifetime $\zeta=\zeta(\omega)$ :

$$
\forall t<\zeta, \quad \omega(t) \in \mathbb{R}, \quad \forall t \geq \zeta, \quad \omega(t)=\Delta .
$$

Let $\left(X_{t}\right)$ denote the canonical process: $X_{t}(\omega)=\omega(t)$. Let $\left(\mathscr{F}_{t}\right)$ denote its natural filtration and $\mathscr{F}_{\infty}=\sigma\left(\cup_{t} \mathscr{F}_{t}\right)$. For $a \in \mathbb{R}$, we write $T_{\{a\}}$ for the first hitting time of the point $a$ :

$$
T_{\{a\}}=\inf \left\{t>0: X_{t}=a\right\} .
$$

The set of all nonnegative Borel functions on $\mathbb{R}$ will be denoted by $\mathscr{B}_{+}(\mathbb{R})$.

Let $\left(\mathbf{P}_{x}: x \in \mathbb{R}\right)$ denote the laws on $\mathbb{D}$ of a right Markov process. We assume that the transition kernels have jointly measurable densities $p_{t}(x, y)$ with respect to a reference measure $\mu(d y)$ :

$$
\mathbf{P}_{x}\left(X_{t} \in d y\right)=p_{t}(x, y) \mu(d y) .
$$

We define

$$
u_{q}(x, y)=\int_{0}^{\infty} e^{-q t} p_{t}(x, y) d t, \quad q \geq 0,
$$

which are resolvent densities if they are finite. We also assume that there exists a local time $\left(L_{t}^{x}\right)$ such that

$$
\int_{0}^{t} f\left(X_{s}\right) d s=\int f(y) L_{t}^{y} \mu(d y), \quad t>0, f \in \mathscr{B}_{+}(\mathbb{R})
$$

holds with $\mathbf{P}_{x}$-probability one for any $x \in \mathbb{R}$.

2.1. Feynman-Kac Formula for Transient Markov Processes. In this section, we prove Feynman-Kac formula for transient Markov processes. We assume the following conditions:

(i) the process is transient;

(ii) $u_{0}(x, y)<\infty$ for any $x, y \in \mathbb{R}$ with $x \neq 0$ or $y \neq 0$.

Note that $u_{0}(0,0)$ may be infinite. We note that

$$
\mathbf{P}_{x}\left(\forall y \in \mathbb{R}, L_{\infty}^{y}<\infty\right)=1 \quad \text { for any } x \in \mathbb{R} .
$$

By formula (5), it is easy to see that

$$
\mathbf{P}_{x}\left[L_{\infty}^{y}\right]=u_{0}(x, y), \quad x \in \mathbb{R}, y \in \mathbb{R} \backslash\{0\} .
$$

We will prove a version of Feynman-Kac formulae following Marcus-Rosen's book [16] where it is assumed that $u_{0}(0,0)<$ $\infty$.

For $t \geq 0$ and $x_{1}, x_{2}, \ldots, x_{n} \in \mathbb{R} \backslash\{0\}$, we set

$$
J_{t}(\mathbf{x})=\int_{t}^{\infty} d L_{t_{1}}^{x_{1}} \int_{t_{1}}^{\infty} d L_{t_{2}}^{x_{2}} \cdots \int_{t_{n-1}}^{\infty} d L_{t_{n}}^{x_{n}}
$$

where $\mathbf{x}=\left(x_{1}, \ldots, x_{n}\right)$.

Theorem 1 (Kac's moment formula). Let $x_{0} \in \mathbb{R}$ and $x_{1}, x_{2}, \ldots, x_{n} \in \mathbb{R} \backslash\{0\}$. Then we has

$$
\mathbf{P}_{x_{0}}\left[J_{0}(\mathbf{x})\right]=u_{0}\left(x_{0}, x_{1}\right) u_{0}\left(x_{1}, x_{2}\right) \cdots u_{0}\left(x_{n-1}, x_{n}\right) .
$$

The proof is essentially the same to that of $[16$, Theorem 2.5.3], but we give it for completeness of the paper.

Proof. Note that

$$
J_{0}(\mathbf{x})=\int_{0}^{\infty} J_{t}\left(\mathbf{x}^{\prime}\right) d L_{t}^{x_{1}}
$$

where $\mathbf{x}^{\prime}=\left(x_{2}, \ldots, x_{n}\right)$. Denote $\tau_{l}^{x_{1}}=\inf \left\{t>0 ; L_{t}^{x_{1}}>l\right\}$. Since $J_{t}\left(\mathbf{x}^{\prime}\right)=J_{0}\left(\mathbf{x}^{\prime}\right) \circ \theta_{t}$, the strong Markov property yields that

$$
\begin{aligned}
\mathbf{P}_{x_{0}}\left[J_{0}(\mathbf{x})\right] & =\mathbf{P}_{x_{0}}\left[\int_{0}^{\infty} J_{0}\left(\mathbf{x}^{\prime}\right) \circ \theta_{\tau_{l}^{x_{1}}} 1_{\left\{\tau_{l}^{x_{1}}<\infty\right\}} d l\right] \\
& =\mathbf{P}_{x_{0}}\left[\int_{0}^{\infty} 1_{\left\{\tau_{l}^{x_{1}}<\infty\right\}} d l\right] \mathbf{P}_{x_{1}}\left[J_{0}\left(\mathbf{x}^{\prime}\right)\right] \\
& =\mathbf{P}_{x_{0}}\left[L_{\infty}^{x_{1}}\right] \mathbf{P}_{x_{1}}\left[J_{0}\left(\mathbf{x}^{\prime}\right)\right] .
\end{aligned}
$$

This yields (9) from (7).

Theorem 2 (Feynman-Kac formula). Let $x_{1}, \ldots, x_{n} \in \mathbb{R} \backslash\{0\}$. Set

$$
\begin{gathered}
\Sigma=\left(\begin{array}{ccc}
u_{0}\left(x_{1}, x_{1}\right) & \cdots & u_{0}\left(x_{1}, x_{n}\right) \\
\vdots & \ddots & \vdots \\
u_{0}\left(x_{n}, x_{1}\right) & \cdots & u_{0}\left(x_{n}, x_{n}\right)
\end{array}\right), \\
\Sigma^{0}=\left(\begin{array}{ccc}
u_{0}\left(0, x_{1}\right) & \cdots & u_{0}\left(0, x_{n}\right) \\
\vdots & \ddots & \vdots \\
u_{0}\left(0, x_{1}\right) & \cdots & u_{0}\left(0, x_{n}\right)
\end{array}\right) .
\end{gathered}
$$

Then, for any diagonal matrix $\Lambda=\left(\lambda_{i} \delta_{i, j}\right)_{i, j=1}^{n}$ with nonnegative entries, we have

$$
\mathbf{P}_{0}\left[\exp \left\{-\sum_{i=1}^{n} \lambda_{i} L_{\infty}^{x_{i}}\right\}\right]=\frac{\operatorname{det}\left(I+\left(\Sigma-\Sigma^{0}\right) \Lambda\right)}{\operatorname{det}(I+\Sigma \Lambda)} .
$$

The proof is almost parallel to that of [16, Lemma 2.6.2], but we give it for completeness of the paper. 
Proof. Let $\lambda_{1}, \ldots, \lambda_{n} \in \mathbb{R}$. For $k \in \mathbb{N}$, we have

$$
\begin{aligned}
& \mathbf{P}_{0}\left[\left(\sum_{j=1}^{n} \lambda_{j} L_{\infty}^{x_{j}}\right)^{k}\right] \\
& =\sum_{j_{1}, \ldots, j_{k}=1}^{n} \lambda_{j_{1}} \cdots \lambda_{j_{k}} \mathbf{P}_{0}\left[L_{\infty}^{x_{j_{1}}} \cdots L_{\infty}^{x_{j_{k}}}\right] \\
& =k ! \sum_{j_{1}, \ldots, j_{k}=1}^{n} \lambda_{j_{1}} \cdots \lambda_{j_{k}} \mathbf{P}_{0}\left[J_{0}\left(x_{j_{1}}, \ldots, x_{j_{k}}\right)\right]
\end{aligned}
$$

It follows from Theorem 1 that

$$
\begin{aligned}
(15)= & k ! \sum_{j_{1}, \ldots, j_{k}=1}^{n} u_{0}\left(0, x_{j_{1}}\right) \lambda_{j_{1}} \\
& \cdot u_{0}\left(x_{j_{1}}, x_{j_{2}}\right) \lambda_{j_{2}} \cdots u_{0}\left(x_{j_{k-1}}, x_{j_{k}}\right) \lambda_{j_{k}} \\
= & k !\left\{(\tilde{\Sigma} \widetilde{\Lambda})^{k} \mathbf{1}\right\}_{0},
\end{aligned}
$$

where $\mathbf{1}={ }^{\top}(1, \ldots, 1),\{\mathbf{v}\}_{0}=v_{0}$ for $\mathbf{v}={ }^{\top}\left(v_{0}, v_{1}, \ldots, v_{n}\right)$,

$$
\begin{gathered}
\tilde{\Sigma}=\left(\begin{array}{cccc}
0 & u_{0}\left(0, x_{1}\right) & \cdots & u_{0}\left(0, x_{n}\right) \\
0 & u_{0}\left(x_{1}, x_{1}\right) & \cdots & u_{0}\left(x_{1}, x_{n}\right) \\
\vdots & \vdots & \ddots & \vdots \\
0 & u_{0}\left(x_{n}, x_{1}\right) & \cdots & u_{0}\left(x_{n}, x_{n}\right)
\end{array}\right), \\
\widetilde{\Lambda}=\left(\begin{array}{cccc}
0 & 0 & \cdots & 0 \\
0 & \lambda_{1} & \cdots & 0 \\
\vdots & \vdots & \ddots & \vdots \\
0 & 0 & \cdots & \lambda_{n}
\end{array}\right)
\end{gathered}
$$

Hence, for all $\lambda_{1}, \ldots, \lambda_{n} \in \mathbb{R}$ such that $\left|\lambda_{i}\right|$ 's are small enough, we have

$$
\begin{aligned}
\mathbf{P}_{0}\left[\exp \left\{\sum_{i=1}^{n} \lambda_{i} L_{\infty}^{x_{i}}\right\}\right] \\
=\sum_{k=0}^{\infty}\left\{(\widetilde{\Sigma} \widetilde{\Lambda})^{k} \mathbf{1}\right\}_{0}=\left\{(I-\widetilde{\Sigma} \widetilde{\Lambda})^{-1} \mathbf{1}\right\}_{0} .
\end{aligned}
$$

By Cramer's formula, we obtain

$$
\begin{aligned}
\left\{(I-\widetilde{\Sigma} \widetilde{\Lambda})^{-1} \mathbf{1}\right\}_{0} \\
\quad=\frac{\operatorname{det}\left((I-\widetilde{\Sigma} \widetilde{\Lambda})^{(1)}\right)}{\operatorname{det}(I-\widetilde{\Sigma} \widetilde{\Lambda})}=\frac{\operatorname{det}\left(I-\left(\Sigma-\Sigma^{0}\right) \Lambda\right)}{\operatorname{det}(I-\Sigma \Lambda)} .
\end{aligned}
$$

Here, for a matrix $A$, we denote by $A^{(1)}$ the matrix which is obtained by replacing each entry in the first column of $A$ by number 1 . Since $\Sigma$ is nonnegative definite, we obtain the desired result (13) by analytic continuation.
Remark 3. Eisenbaum et al. [6] have proved an analogue of Ray-Knight theorem for the total local time of a symmetric Lévy process killed at an independent exponential time. We may say that the key to the proof is that $\Sigma-\Sigma^{0}$ is a constant matrix which is positive definite. The difficulty in the case of the $h$-path process of a symmetric Lévy process is that the matrix $\Sigma-\Sigma^{0}$ no longer has such a nice property.

2.2. Feynman-Kac Formula for Markovian Bridges. In this section, we show Feynman-Kac formula for Markovian bridges. For this, we recall several theorems for Markovian bridges from Fitzsimmons et al. [15]. See [15] for details.

For $t>0, x, y \in \mathbb{R}$, let $\mathbf{P}_{x, y}^{t}$ denote the bridge law, which serves as a version of the regular conditional distribution for $\left\{X_{s} ; 0 \leq s \leq t\right\}$ under $\mathbf{P}_{x}$ given $X_{t-}=y$. In this section, we assume the following condition:

(i) $0<p_{t}(x, y)<\infty$ for any $t>0, x, y \in \mathbb{R}$.

We also assume that there exists a local time $\left(L_{t}^{x}\right)$ such that

$$
\int_{0}^{s} f\left(X_{u}\right) d u=\int f(y) L_{s}^{y} \mu(d y), \quad 0 \leq s \leq t,
$$

$$
f \in \mathscr{B}_{+}(\mathbb{R})
$$

holds with $\mathbf{P}_{x, y}^{t}$-probability one for any $t>0$ and $x, y \in \mathbb{R}$.

Theorem 4 (see $[15$, Lemma 1]). Let $t>0, x, y, z \in \mathbb{R}$. Then one has

$$
\mathbf{P}_{x, y}^{t}\left[\int_{0}^{t} f\left(s, X_{s}\right) d L_{s}^{z}\right]=\int_{0}^{t} d s \frac{p_{s}(x, z) p_{t-s}(z, y)}{p_{t}(x, y)} f(s, z)
$$

for any nonnegative Borel function $f$.

We will also use the following conditioning formula.

Theorem 5 (see [15, Proposition 3]). Let $t>0, x, y, z \in \mathbb{R}$. Then one has

$$
\begin{aligned}
\mathbf{P}_{x, y}^{t}[ & {\left[\int_{0}^{t} f\left(s, X_{s}\right) H_{s} d L_{s}^{z}\right] } \\
& =\mathbf{P}_{x, y}^{t}\left[\int_{0}^{t} f(s, z) \mathbf{P}_{x, z}^{s}\left[H_{s}\right] d L_{s}^{z}\right]
\end{aligned}
$$

for any nonnegative Borel function $f$ and any nonnegative predictable process $H_{s}$.

For $s \geq 0$ and $z_{1}, \ldots, z_{n} \in \mathbb{R}$, we define

$$
H_{s}\left(\mathbf{z}^{(n)}\right)=\int_{0}^{s} d L_{s_{n}}^{z_{n}} \int_{0}^{s_{n}} d L_{s_{n-1}}^{z_{n-1}} \ldots \int_{0}^{s_{2}} d L_{s_{1}}^{z_{1}}
$$

where $\mathbf{z}^{(n)}=\left(z_{1}, \ldots, z_{n}\right)$. The following theorem is a version of Kac's moment formulae. 
Theorem 6. For any $q>0, n \in \mathbb{N}$ and for any $z_{1}, \ldots, z_{n} \in \mathbb{R}$, one has

$$
\begin{gathered}
\int_{0}^{\infty} e^{-q t} p_{t}(x, y) \mathbf{P}_{x, y}^{t}\left[H_{t}\left(\mathbf{z}^{(n)}\right)\right] d t \\
=u_{q}\left(x, z_{1}\right) \cdot \prod_{j=1}^{n-1} u_{q}\left(z_{j}, z_{j+1}\right) \\
\cdot u_{q}\left(z_{n}, y\right) .
\end{gathered}
$$

Proof. Let us prove the claim by induction. For $n=1$, the assertion follows from Theorem 4 . Suppose that formula (24) holds for a given $n \geq 2$. Note that

$$
H_{t}\left(\mathbf{z}^{(n+1)}\right)=\int_{0}^{t} H_{s}\left(\mathbf{z}^{(n)}\right) d L_{s}^{z_{n+1}}
$$

Since $H_{s}\left(\mathbf{z}^{(n)}\right)$ is a nonnegative predictable process, Theorems 5 and 4 show that

$$
\begin{aligned}
\mathbf{P}_{x, y}^{t}[ & \left.H_{t}\left(\mathbf{z}^{(n+1)}\right)\right] \\
& =\int_{0}^{t} d s \frac{p_{s}\left(x, z_{n+1}\right) p_{t-s}\left(z_{n+1}, y\right)}{p_{t}(x, y)} \mathbf{P}_{x, z_{n+1}}^{t}\left[H_{s}\left(z^{(n)}\right)\right] .
\end{aligned}
$$

Hence, we obtain

$$
\begin{aligned}
\int_{0}^{\infty} e^{-q t} & p_{t}(x, y) \mathbf{P}_{x, y}^{t}\left[H_{t}\left(z^{(n+1)}\right)\right] d t \\
= & \int_{0}^{\infty} e^{-q t} d t \\
& \times \int_{0}^{t} p_{s}\left(x, z_{n+1}\right) p_{t-s}\left(z_{n+1}, y\right) \mathbf{P}_{x, z_{n+1}}^{s}\left[H_{s}\left(\mathbf{z}^{(n)}\right)\right] d s \\
= & \int_{0}^{\infty} e^{-q s} p_{s}\left(x, z_{n+1}\right) \mathbf{P}_{x, z_{n+1}}^{s}\left[H_{s}\left(\mathbf{z}^{(n)}\right)\right] d s \\
& \times \int_{0}^{\infty} e^{-q t} p_{t}\left(z_{n+1}, y\right) d t \\
= & u_{q}\left(x, z_{1}\right) \cdot \prod_{j=1}^{n-1} u_{q}\left(z_{j}, z_{j+1}\right) \\
& \cdot u_{q}\left(z_{n}, z_{n+1}\right) \cdot u_{q}\left(z_{n+1}, y\right),
\end{aligned}
$$

by the assumption of the induction. Now we have proved that formula (24) is valid also for $n+1$, which completes the proof.

The following theorem is a version of Feynman-Kac formulae.

Theorem 7. Let $z_{1}=0, z_{2}, \ldots, z_{n} \in \mathbb{R}$ and let $\lambda_{1}, \ldots, \lambda_{n} \geq 0$. Suppose that

$$
u_{q}\left(z_{i}, z_{j}\right)<\infty, \quad q>0, i, j=1, \ldots, n .
$$

Let $\Sigma^{(q)}$ be the matrix with elements $\Sigma_{i, j}^{(q)}=u_{q}\left(z_{i}, z_{j}\right)$. Then, for any diagonal matrix $\Lambda=\left(\lambda_{i} \delta_{i, j}\right)_{i, j=1}^{n}$ with nonnegative entries, one has

$$
\begin{gathered}
\int_{0}^{\infty} e^{-q t} p_{t}(0,0) \mathbf{P}_{0,0}^{t}\left[e^{-\sum_{j=1}^{n} \lambda_{j} L_{t}^{z_{j}}}\right] d t \\
=\left\{\left(I+\Sigma^{(q)} \Lambda\right)^{-1} \Sigma^{(q)}\right\}_{1,1} .
\end{gathered}
$$

Proof. We have

$$
\begin{aligned}
\int_{0}^{\infty} e^{-q t} p_{t}(0,0) \mathbf{P}_{0,0}^{t}\left[\left(\sum_{j=1}^{n} \lambda_{j} L_{t}^{z_{j}}\right)^{k}\right] d t \\
=k ! \sum_{j_{1}, \ldots, j_{k}=1}^{n} \lambda_{j_{k}} \cdots \lambda_{j_{1}} \\
\quad \times \int_{0}^{\infty} e^{-q t} p_{t}(0,0) \mathbf{P}_{0,0}^{t}\left[H_{t}\left(z_{j_{k}}, \ldots, z_{j_{1}}\right)\right] d t
\end{aligned}
$$

Using Theorem 6 , we see that the above quantity is equal to

$$
\begin{aligned}
k ! & \sum_{j_{1}, \ldots, j_{k}=1}^{n} u_{q}\left(z_{1}, z_{j_{1}}\right) \lambda_{j_{1}} \\
& \cdot \prod_{i=1}^{k-1} u_{q}\left(z_{j_{i}}, z_{j_{i+1}}\right) \lambda_{j_{i+1}} \cdot u_{q}\left(z_{j_{k}}, z_{1}\right),
\end{aligned}
$$

which amounts to $k !\left\{\left(\Sigma^{(q)} \Lambda\right)^{k} \Sigma^{(q)}\right\}_{1,1}$. Hence, for all $\lambda_{1}, \ldots$, $\lambda_{n}>0$ sufficiently small, we obtain

$$
\begin{gathered}
\int_{0}^{\infty} e^{-q t} p_{t}(0,0) \mathbf{P}_{0,0}^{t}\left[\exp \left\{\sum_{j=1}^{n} \lambda_{j} L_{t}^{z_{j}}\right\}\right] d t \\
=u_{q}(0,0)+\sum_{k=1}^{\infty}\left\{\left(\Sigma^{(q)} \Lambda\right)^{k} \Sigma^{(q)}\right\}_{1,1} \\
=\left\{\left(I-\Sigma^{(q)} \Lambda\right)^{-1} \Sigma^{(q)}\right\}_{1,1} .
\end{gathered}
$$

Since $\Sigma^{(q)}$ is nonnegative definite, we obtain the desired result (29) by analytic continuation.

The following theorem is valid even if

$$
u_{q}\left(0, z_{j}\right)=u_{q}\left(z_{j}, 0\right)=\infty, \quad q>0, j=1, \ldots, n .
$$

Theorem 8. Let $z_{1}, \ldots, z_{n} \in \mathbb{R} \backslash\{0\}$ and let $\lambda_{1}, \ldots, \lambda_{n} \geq 0$. Suppose that

$$
u_{q}\left(z_{i}, z_{j}\right)<\infty, \quad q>0, i, j=1, \ldots, n .
$$

Let $\Sigma^{(q)}$ be the matrix with elements $\Sigma_{i, j}^{(q)}=u_{q}\left(z_{i}, z_{j}\right)$,

$$
\mathbf{u}^{(q)}=\left(\begin{array}{c}
u_{q}\left(0, z_{1}\right) \\
\vdots \\
u_{q}\left(0, z_{n}\right)
\end{array}\right), \quad \mathbf{v}^{(q)}=\left(\begin{array}{c}
u_{q}\left(z_{1}, 0\right) \\
\vdots \\
u_{q}\left(z_{n}, 0\right)
\end{array}\right) \text {, }
$$


and let $\Lambda$ be the matrix with elements $\Lambda_{i, j}=\lambda_{i} \delta_{i, j}$. Then one has

$$
\begin{gathered}
\int_{0}^{\infty} e^{-q t} p_{t}(0,0) \mathbf{P}_{0,0}^{t}\left[1-e^{-\sum_{j=1}^{n} \lambda_{j} L_{t}^{z_{j}}}\right] d t \\
=^{\top} \mathbf{u}^{(q)} \Lambda\left(I+\Sigma^{(q)} \Lambda\right)^{-1} \mathbf{v}^{(q)} .
\end{gathered}
$$

Proof. Using Theorem 6, we see that

$$
\begin{gathered}
\int_{0}^{\infty} e^{-q t} p_{t}(0,0) \mathbf{P}_{0,0}^{t}\left[\left(\sum_{j=1}^{n} \lambda_{j} L_{t}^{z_{j}}\right)^{k}\right] d t \\
=k ! \sum_{j_{1}, \ldots, j_{k}=1}^{n} u_{q}\left(0, z_{j_{1}}\right) \lambda_{j_{1}} \cdot \prod_{i=1}^{k-1} u_{q}\left(z_{j_{i}}, z_{j_{i+1}}\right) \lambda_{j_{i+1}} \\
\cdot u_{q}\left(z_{j_{k}}, 0\right) .
\end{gathered}
$$

Hence, we obtain

$$
\begin{gathered}
\int_{0}^{\infty} e^{-q t} p_{t}(0,0) \mathbf{P}_{0,0}^{t}\left[\exp \left\{\sum_{j=1}^{n} \lambda_{j} L_{t}^{z_{j}}\right\}-1\right] d t \\
=\sum_{k=1}^{\infty}\left\{{ }^{\top} \mathbf{u}^{(q)} \Lambda\left(\Sigma^{(q)} \Lambda\right)^{k-1} \mathbf{v}^{(q)}\right\} \\
={ }^{\top} \mathbf{u}^{(q)} \Lambda\left(I-\Sigma^{(q)} \Lambda\right)^{-1} \mathbf{v}^{(q)} .
\end{gathered}
$$

The rest of the proof is now obvious.

\section{Preliminaries: Squared Bessel Processes and Generalized Excursion Measures}

In this section, we recall squared Bessel processes and generalized excursion measures.

First, we introduce several notations about squared Bessel processes, for which we follow [3, XI.1]. For $\delta \geq 0$, let $\left(\mathbf{Q}_{z}^{\delta}\right.$ : $z \geq 0$ ) denote the law of the $\delta$-dimensional squared Bessel process where the origin is a trap when $\delta=0$. Then the Laplace transform of a one-dimensional marginal is given by

$$
\mathbf{Q}_{z}^{\delta}\left[\exp \left\{-\lambda X_{t}\right\}\right]=\frac{1}{(1+2 \lambda t)^{\delta / 2}} \exp \left\{-\frac{\lambda z}{1+2 \lambda t}\right\}
$$

We may obtain the transition kernels $\mathbf{Q}_{z}^{\delta}\left(X_{t} \in d w\right)$ by the Laplace inversion.

(i) For $\delta>0$ and $z>0$, we have

$$
\begin{aligned}
\mathbf{Q}_{z}^{\delta} & \left(X_{t} \in d w\right) \\
& =\frac{1}{2 t}\left(\frac{w}{z}\right)^{(1 / 2)(\delta / 2-1)} \exp \left\{-\frac{z+w}{2 t}\right\} I_{\delta / 2-1}\left(\frac{\sqrt{z w}}{t}\right) d w
\end{aligned}
$$

where $I_{v}$ stands for the modified Bessel function of order $v$. (ii) For $\delta>0$ and $z=0$, we have

$$
\mathbf{Q}_{0}^{\delta}\left(X_{t} \in d w\right)=\frac{1}{(2 t)^{\delta / 2} \Gamma(\delta / 2)} w^{\delta / 2-1} \exp \left\{-\frac{w}{2 t}\right\} d w
$$

where $\Gamma$ stands for the gamma function.

(iii) For $\delta=0$ and $z \geq 0$, we have

$$
\begin{aligned}
\mathbf{Q}_{z}^{0}\left(X_{t} \in d w\right)= & \exp \left\{-\frac{z}{2 t}\right\} \delta_{0}(d w) \\
& +\frac{1}{2 t}\left(\frac{w}{z}\right)^{-1 / 2} \exp \left\{-\frac{z+w}{2 t}\right\} \\
& \times I_{1}\left(\frac{\sqrt{z w}}{t}\right) d w .
\end{aligned}
$$

The squared Bessel process satisfies the scaling property: for $\delta \geq 0, z \geq 0$, and $c>0$, it holds that

$$
\left(c X_{t / c}\right) \text { under } \mathbf{Q}_{z / c}^{\delta} \stackrel{\text { law }}{=}\left(X_{t}\right) \text { under } \mathbf{Q}_{z}^{\delta} .
$$

Second, we recall the notion of the generalized excursion measure. By formula (39), we have

$$
\mathbf{Q}_{0}^{4}\left[\frac{1}{X_{s+t}^{2}} ; X_{s+t} \in B\right]=\mathbf{Q}_{0}^{4}\left[\frac{1}{X_{s}^{2}} \cdot \mathbf{Q}_{X_{s}}^{0}\left(X_{t} \in B\right)\right]
$$

for $s, t>0$ and $B \in \mathscr{B}([0, \infty))$. If we put $\mu_{t}(d x)=$ $\left(1 / x^{2}\right) \mathbf{Q}_{0}^{4}\left(X_{t} \in d x\right)$, we have

$$
\mu_{s+t}(B)=\int \mu_{s}(d x) \mathbf{Q}_{x}^{0}\left(X_{t} \in B\right)
$$

This shows that the family of laws $\left\{\mu_{t}: t>0\right\}$ is an entrance law for $\left\{\mathbf{Q}_{x}^{0}: x>0\right\}$. In fact, there exists a unique $\sigma$-finite measure $\mathbf{n}^{(0)}$ on $\mathbb{D}$ such that

$$
\begin{aligned}
\mathbf{n}^{(0)}\left(X_{t_{1}} \in B_{1}, \ldots, X_{t_{n}} \in B_{n}\right) \\
=\int_{B_{1}} \mu_{t_{1}}\left(d x_{1}\right) \int_{B_{2}} \mathbf{Q}_{x}^{0}\left(X_{t_{2}-t_{1}} \in d x_{2}\right) \\
\quad \ldots \int_{B_{n}} \mathbf{Q}_{x}^{0}\left(X_{t_{n}-t_{n-1}} \in d x_{n}\right)
\end{aligned}
$$

for $0<t_{1}<\cdots<t_{n}$ and $B_{1}, \ldots, B_{n} \in \mathscr{B}([0, \infty))$. Note that, to construct such a measure $\mathbf{n}^{(0)}$, we can not appeal to Kolmogorov's extension theorem, because the entrance laws have infinite total mass. However, we can actually construct $\mathbf{n}^{(0)}$ via the agreement formula (see Pitman-Yor [17, Cor. 3] with $\delta=4$ ), or via the time change of a Brownian excursion (see Fitzsimmons-Yano [18, Theorem 2.5] with change of scales). We may call $\mathbf{n}^{(0)}$ the generalized excursion measure for the squared Bessel process of dimension 0 . See the references above for several characteristic formulae of $\mathbf{n}^{(0)}$. 


\section{Symmetric Lévy Processes}

Let us confine ourselves to one-dimensional symmetric Lévy processes. We recall general facts and state several results from [7].

In what follows, we assume that $\left(\mathbf{P}_{x}\right)$ is the law of a one-dimensional conservative Lévy process. Throughout the present paper, we assume the following conditions, which will be referred to as (A), are satisfied:

(i) the process is symmetric;

(ii) the origin (and, consequently, any point) is regular for itself;

(iii) the process is not a compound Poisson.

Under the condition (A), we have the following. The characteristic exponent is given by

$$
\theta(\lambda):=-\log \mathbf{P}_{0}\left[e^{i \lambda X_{1}}\right]=v \lambda^{2}+2 \int_{0}^{\infty}(1-\cos \lambda x) v(d x),
$$

for some $v \geq 0$ and some positive Radon measure $v$ on $(0, \infty)$ such that

$$
\int_{(0, \infty)} \min \left\{x^{2}, 1\right\} v(d x)<\infty
$$

The reference measure is $\mu(d x)=d x$ and we have

$$
\begin{aligned}
& p_{t}(x, y)=p_{t}(y-x)=\frac{1}{\pi} \int_{0}^{\infty}(\cos \lambda(y-x)) e^{-t \theta(\lambda)} d \lambda \\
& u_{q}(x, y)=u_{q}(y-x)=\frac{1}{\pi} \int_{0}^{\infty} \frac{\cos \lambda(y-x)}{q+\theta(\lambda)} d \lambda
\end{aligned}
$$

There exists a local time $\left(L_{t}^{x}\right)$ such that

$$
\int_{0}^{t} f\left(X_{s}\right) d s=\int f(y) L_{t}^{y} d y, \quad f \in \mathscr{B}_{+}(\mathbb{R}),
$$

with $\mathbf{P}_{x}$-probability one for any $x \in \mathbb{R}$. Then it holds that

$$
\mathbf{P}_{x}\left[\int_{0}^{\infty} e^{-q s} d L_{s}^{y}\right]=u_{q}(y-x), \quad x, y \in \mathbb{R} .
$$

Let $\mathbf{n}$ denote the excursion measure associated to the local time $L_{t}^{0}$. We denote by $\left(\mathbf{P}_{x}^{0}: x \in \mathbb{R} \backslash\{0\}\right)$ the law of the process killed upon hitting the origin; that is,

$$
\begin{array}{r}
\mathbf{P}_{x}^{0}(A ; \zeta>t)=\mathbf{P}_{x}\left(A ; T_{\{0\}}>t\right), \quad x \in \mathbb{R} \backslash\{0\}, \\
t>0, A \in \mathscr{F}_{t} .
\end{array}
$$

Then the excursion measure $\mathbf{n}$ satisfies the Markov property in the following sense: for any $t>0$ and for any nonnegative $\mathscr{F}_{t}$-measurable functional $Z_{t}$ and for any nonnegative $\mathscr{F}_{\infty}{ }^{-}$ measurable functional $F$, it holds that

$$
\mathbf{n}\left[Z_{t} F\left(X_{t+\cdot}\right)\right]=\int \mathbf{n}\left[Z_{t} ; X_{t} \in d x\right] \mathbf{P}_{x}^{0}[F(X)] .
$$

We need the following additional conditions:

(R) the process is recurrent;

(T) the function $\theta(\lambda)$ is nondecreasing in $\lambda>\lambda_{0}$ for some $\lambda_{0}>0$.

Under the condition (A), the condition ( $\mathbf{R})$ is equivalent to

$$
\int_{0}^{\infty} \frac{d \lambda}{\theta(\lambda)}=\infty
$$

All of the conditions (A), (R), and (T) are obviously satisfied if the process is a symmetric stable Lévy process of index $\alpha \epsilon$ $(1,2]$ :

$$
\theta(\lambda)=|\lambda|^{\alpha}
$$

In what follows, we assume, as well as the condition (A), that the conditions $(\mathbf{R})$ and $(\mathbf{T})$ are also satisfied.

The Laplace transform of the law of $T_{\{0\}}$ is given by

$$
\mathbf{P}_{z}\left[e^{-q T_{\{0\}}}\right]=\frac{u_{q}(z)}{u_{q}(0)},
$$

see, for example, [19, pp. 64]. It is easy to see that the entrance law has the space density:

$$
\rho(t, x)=\frac{\mathbf{n}\left(X_{t} \in d x\right)}{d x} .
$$

In view of [7, Theorem 2.10], the law of the hitting time $T_{\{0\}}$ is absolutely continuous relative to the Lebesgue measure $d t$ and the time density coincides with the space density of the entrance law:

$$
\rho_{x}(t)=\frac{\mathbf{P}_{x}\left(T_{\{0\}} \in d t\right)}{d t}=\rho(t, x) .
$$

4.1. Absolute Continuity of the Law of the Inverse Local Time. Let $\tau(l)$ denote the inverse local time at the origin:

$$
\tau(l)=\inf \left\{t>0 ; L_{t}^{0}>l\right\} .
$$

We prove the absolute continuity of the law of inverse local time. Note that $\tau(l)$ is a subordinator such that

$$
\mathbf{P}_{0}\left[e^{-q \tau(l)}\right]=e^{-l / u_{q}(0)},
$$

see, for example, [19, pp. 131].

Lemma 9. For fixed $l>0$, the law of $\tau(l)$ under $\mathbf{P}_{0}$ has a density $\gamma_{l}(t)$

$$
\mathbf{P}_{0}(\tau(l) \in d t)=\gamma_{l}(t) d t
$$

Furthermore, $\gamma_{l}(t)$ may be chosen to be jointly continuous in $(l, t) \in(0, \infty) \times(0, \infty)$.

Proof. Following [7, Sec. 3.3], we define a positive Borel measure $\sigma$ on $[0, \infty)$ as

$$
\sigma(A)=\frac{1}{\pi} \int_{0}^{\infty} 1_{A}(\theta(\lambda)) d \lambda, \quad A \in \mathscr{B}([0, \infty)) .
$$


Then we have $u_{q}(0)=\int_{[0, \infty)}(\sigma(d \xi) /(q+\xi))$ for $q>0$, and hence there exists a Radon measure $\sigma^{*}$ on $[0, \infty)$ with $\int_{[0, \infty)}\left(\sigma^{*}(d \xi) /(1+\xi)\right)<\infty$ such that

$$
\frac{1}{q u_{q}(0)}=\int_{[0, \infty)} \frac{1}{q+\xi} \sigma^{*}(d \xi), \quad q>0 .
$$

Hence, the Laplace exponent $1 / u_{q}(0)$ may be represented as

$$
\frac{1}{u_{q}(0)}=\int_{0}^{\infty}\left(1-e^{-q u}\right) v(u) d u
$$

where $v(u)=\int_{(0, \infty)} e^{-u \xi} \xi \sigma^{*}(d \xi)$. Since $\int_{0}^{\infty}\left(1 \wedge u^{2}\right) v(u) d u<$ $\infty$, we may appeal to analytic continuation of both sides of formula (61) and obtain

$$
\mathbf{P}_{0}\left[e^{i \lambda \tau(l)}\right]=\exp \left\{l \int_{0}^{\infty}\left(e^{i \lambda u}-1\right) v(u) d u\right\}
$$

Following [20, Theorem 3.1], we may invert the Fourier transform of the law of $\tau(l)$ and obtain the desired result.

4.2. h-Paths of Symmetric Lévy Processes. We follow [7] for the notations concerning $h$-paths of symmetric Lévy processes. For the interpretation of the $h$-paths as some kind of conditioning, see [10].

We define

$$
\begin{aligned}
h(x) & =\lim _{q \rightarrow 0+}\left\{u_{q}(0)-u_{q}(x)\right\} \\
& =\frac{1}{\pi} \int_{0}^{\infty} \frac{1-\cos \lambda x}{\theta(\lambda)} d \lambda, \quad x \in \mathbb{R} .
\end{aligned}
$$

The second equality follows from (50). Then the function $h$ satisfies the following:

(i) $h(x)$ is continuous;

(ii) $h(0)=0, h(x)>0$ for all $x \in \mathbb{R} \backslash\{0\}$;

(iii) $h(x) \rightarrow \infty$ as $|x| \rightarrow \infty$ (since the condition $(\mathbf{R})$ is satisfied).

See [7, Lemma 4.2] for the proof. Moreover, the function $h$ is harmonic with respect to the killed process:

$$
\begin{gathered}
\mathbf{P}_{x}^{0}\left[h\left(X_{t}\right)\right]=h(x) \quad \text { if } x \in \mathbb{R} \backslash\{0\}, t>0, \\
\mathbf{n}\left[h\left(X_{t}\right)\right]=1 \quad \text { if } t>0 .
\end{gathered}
$$

See [7, Theorems 1.1 and 1.2] for the proof. We define the $h$ path process $\left(\mathbf{P}_{x}^{h}: x \in \mathbb{R}\right)$ by the following local equivalence relations:

$$
d \mathbf{P}_{\left.x\right|_{\mathscr{F}_{t}} ^{h}}^{h}= \begin{cases}\left.\frac{h\left(X_{t}\right)}{h(x)} d \mathbf{P}_{x}^{0}\right|_{\mathscr{F}_{t}} & \text { if } x \in \mathbb{R} \backslash\{0\}, \\ \left.h\left(X_{t}\right) d \mathbf{n}\right|_{\mathscr{F}_{t}} & \text { if } x=0 .\end{cases}
$$

Remark that, from the strong Markov properties of $\left(X_{t}\right)$ under $\mathbf{P}_{x}^{0}$ and $\mathbf{n}$, the family $\left\{\left.\mathbf{P}_{x}^{h}\right|_{\mathscr{F}_{t}} ; t \geq 0\right\}$ is consistent, and hence the probability measure $\mathbf{P}_{x}^{h}$ is well defined.
The $h$-path process is then symmetric; more precisely, the transition kernel has a symmetric density $p_{t}^{h}(x, y)$ with respect to the measure $h(y)^{2} d y$. Here the density $p_{t}^{h}(x, y)$ is given by

$$
\begin{gathered}
p_{t}^{h}(x, y)=\frac{1}{h(x) h(y)}\left\{p_{t}(y-x)-\frac{p_{t}(x) p_{t}(y)}{p_{t}(0)}\right\} \\
\text { if } x, y \in \mathbb{R} \backslash\{0\}, \\
p_{t}^{h}(x, 0)=p_{t}^{h}(0, x)=\frac{\rho(t, x)}{h(x)} \\
\text { if } x \in \mathbb{R} \backslash\{0\}, \\
p_{t}^{h}(0,0)=\int_{(0, \infty)} e^{-t \xi} \xi \sigma^{*}(d \xi) .
\end{gathered}
$$

By (65), we see that $p_{t}^{h}(0,0)$ is characterized by

$$
\int_{0}^{\infty}\left(1-e^{-q t}\right) p_{t}^{h}(0,0) d t=\frac{1}{u_{q}(0)}, \quad q>0 .
$$

See [7, Section 5] for the details. The $h$-path process also satisfies the following conditions:

(i) the process is conservative;

(ii) any point is regular for itself;

(iii) the process is transient (since the condition $(\mathbf{T})$ is satisfied).

We can easily prove regularity of any point by the local equivalence (69). See [7, Theorem 1.4] for the proof of transience.

The resolvent density of the $h$-path process with respect to $h(y)^{2} d y$ is given by

$$
\begin{gathered}
u_{q}^{h}(x, y)=\frac{1}{h(x) h(y)}\left\{u_{q}(x-y)-\frac{u_{q}(x) u_{q}(y)}{u_{q}(0)}\right\}, \\
q>0, x, y \in \mathbb{R} \backslash\{0\}, \\
u_{q}^{h}(x, 0)=u_{q}^{h}(0, x)=\frac{1}{h(x)} \cdot \frac{u_{q}(x)}{u_{q}(0)}, \quad q>0, x \in \mathbb{R} \backslash\{0\} .
\end{gathered}
$$

We remark here that, since $\lim _{q \rightarrow \infty} u_{q}(0)=0$, we see, by $(71)$, that

$$
u_{q}^{h}(0,0)=\infty
$$

The Green function $u_{0}^{h}(x, y)=\lim _{q \rightarrow 0+} u_{q}^{h}(x, y)$ exists and is given by

$$
\begin{gathered}
u_{0}^{h}(x, y)=\frac{h(x)+h(y)-h(x-y)}{h(x) h(y)}, \quad x, y \in \mathbb{R} \backslash\{0\}, \\
u_{0}^{h}(x, 0)=u_{0}^{h}(0, x)=\frac{1}{h(x)}, \quad x \in \mathbb{R} \backslash\{0\} .
\end{gathered}
$$


See [7, Section 5.3] for the proof. Since $u_{0}^{h}(x, y) \geq 0$, we have

$$
h(x)+h(y)-h(x-y) \geq 0, \quad x, y \in \mathbb{R} .
$$

It follows from the local equivalence (69) that there exists a local time $\left(L_{t}^{x}\right)$ such that

$$
\int_{0}^{t} f\left(X_{s}\right) d s=\int f(y) L_{t}^{y} h(y)^{2} d y, \quad t>0, f \in \mathscr{B}_{+}(\mathbb{R})
$$

with $\mathbf{P}_{x}^{h}$-probability one for any $x \in \mathbb{R}$. We have

$$
\mathbf{P}_{x}^{h}\left[L_{\infty}^{y}\right]=u_{0}^{h}(x, y), \quad x \in \mathbb{R}, y \in \mathbb{R} \backslash\{0\} .
$$

Example 10. If the process is the symmetric stable process of index $\alpha \in(1,2]$, then the harmonic function $h(x)$ may be computed as

$$
h(x)=C(\alpha)|x|^{\alpha-1},
$$

where $C(\alpha)$ is given as follows (see [9, Appendix]):

$$
C(\alpha)=\frac{1}{\pi} \int_{0}^{\infty} \frac{1-\cos \lambda}{\lambda^{\alpha}} d \lambda=\frac{1}{2 \Gamma(\alpha) \sin (\pi(\alpha-1) / 2)} .
$$

\section{The Laws of the Total Local Times for $h$-Paths}

In this section, we state and prove our main theorems concerning the laws of the total local times of $h$-paths.

5.1. Laplace Transform Formula for $h$-Paths. In this section, we prove Laplace transform formula for $h$-paths at two levels.

Lemma 11. For $x, y \in \mathbb{R} \backslash\{0\}$ and $\lambda_{1}, \lambda_{2} \geq 0$, one has

$$
\begin{gathered}
\mathbf{P}_{0}^{h}\left[\exp \left\{-\lambda_{1} h(x) L_{\infty}^{x}-\lambda_{2} h(y) L_{\infty}^{y}\right\}\right] \\
=\frac{1+\lambda_{1}+\lambda_{2}+D \lambda_{1} \lambda_{2}}{1+2 \lambda_{1}+2 \lambda_{2}+4 E \lambda_{1} \lambda_{2}},
\end{gathered}
$$

where

$$
\begin{gathered}
D=D(x, y)=h(x-y) \cdot \frac{h(x)+h(y)-h(x-y)}{h(x) h(y)} \geq 0, \\
E=E(x, y)=1-\frac{(h(x)+h(y)-h(x-y))^{2}}{4 h(x) h(y)} \geq 0 .
\end{gathered}
$$

Proof. Let us apply Theorem 2 with

$I+\left(\Sigma-\Sigma^{0}\right) \Lambda$

$=\left(\begin{array}{cc}1+\lambda_{1} & \frac{h(y)-h(x-y)}{h(x)} \lambda_{2} \\ \frac{h(x)-h(x-y)}{h(y)} \lambda_{1} & 1+\lambda_{2}\end{array}\right)$,

$I+\Sigma \Lambda$

$$
=\left(\begin{array}{cc}
1+2 \lambda_{1} & \frac{h(x)+h(y)-h(x-y)}{h(x)} \lambda_{2} \\
\frac{h(x)+h(y)-h(x-y)}{h(y)} \lambda_{1} & 1+2 \lambda_{2}
\end{array}\right) .
$$

Then we obtain (80) by an easy computation.

By (75), we have $D \geq 0$. Since

$$
E=\frac{h(x) h(y)}{4} \operatorname{det}\left(\begin{array}{ll}
u_{0}^{h}(x, x) & u_{0}^{h}(x, y) \\
u_{0}^{h}(y, x) & u_{0}^{h}(y, y)
\end{array}\right),
$$

we obtain $E \geq 0$ by nonnegative definiteness of the above matrix. The proof is now complete.

5.2. The Law of $L_{\infty}^{x}$. Using formula (80), we can determine the law of $L_{\infty}^{x}$; see [16, Example 3.10.5] for the formula in a more general case.

Theorem 12. For any $x \in \mathbb{R} \backslash\{0\}$, one has

$$
\mathbf{P}_{0}^{h}\left(h(x) L_{\infty}^{x} \in d l\right)=\frac{1}{2}\left\{\delta_{0}(d l)+e^{-l / 2} \frac{d l}{2}\right\}
$$

where $\delta_{0}$ stands for the Dirac measure concentrated at 0 . Consequently, one has

$$
\mathbf{P}_{0}^{h}\left(L_{\infty}^{x}=0\right)=\frac{1}{2} .
$$

Proof. Letting $\lambda_{2}=0$ in Lemma 11, we have

$$
\mathbf{P}_{0}^{h}\left[\exp \left\{-\lambda_{1} h(x) L_{\infty}^{x}\right\}\right]=\frac{1+\lambda_{1}}{1+2 \lambda_{1}}=\frac{1}{2}\left(1+\frac{1}{1+2 \lambda_{1}}\right),
$$

which proves the claim.

Remark 13. Since $L_{\infty}^{x}=0$ if and only if $T_{\{x\}}=\infty$, the identity (85) is equivalent to

$$
\mathbf{P}_{0}^{h}\left(T_{\{x\}}=\infty\right)=\frac{1}{2} .
$$

This formula may also be obtained from the following formula (see [9, Proposition 5.10]):

$$
\mathbf{n}\left(T_{\{x\}}<\zeta\right)=\frac{1}{2 h(x)}
$$


Suppose that, in the definition (69), we may replace the fixed time $t$ with the stopping time $T_{\{x\}}$. Then we have

$$
\begin{aligned}
\mathbf{P}_{0}^{h}\left(T_{\{x\}}<\infty\right) & =\mathbf{n}\left[h\left(X_{T_{\{x\}}}\right) ; T_{\{x\}}<\zeta\right] \\
& =h(x) \mathbf{n}\left(T_{\{x\}}<\zeta\right)=\frac{1}{2} .
\end{aligned}
$$

5.3. The Probability That Two Levels Are Attained. Let us discuss the probability that the total local times at two given levels are positive.

Theorem 14. Let $x, y \in \mathbb{R} \backslash\{0\}$ such that $x \neq y$. Then one has $E>0$ and

$$
\begin{gathered}
\mathbf{P}_{0}^{h}\left(L_{\infty}^{x}>0, L_{\infty}^{y}>0\right)=\mathbf{P}_{0}^{h}\left(L_{\infty}^{x}=L_{\infty}^{y}=0\right)=\frac{D}{4 E}, \\
\mathbf{P}_{0}^{h}\left(L_{\infty}^{x}>0, L_{\infty}^{y}=0\right)=\mathbf{P}_{0}^{h}\left(L_{\infty}^{x}=0, L_{\infty}^{y}>0\right)=\frac{1}{2}-\frac{D}{4 E} .
\end{gathered}
$$

Consequently, one has $D \leq 2 E$.

Proof. Letting $\lambda_{1}=\lambda_{2}=\lambda \geq 0$ in formula (80), we have

$$
\mathbf{P}_{0}^{h}\left[\exp \left\{-\lambda h(x) L_{\infty}^{x}-\lambda h(y) L_{\infty}^{y}\right\}\right]=\frac{1+2 \lambda+D \lambda^{2}}{1+4 \lambda+4 E \lambda^{2}}
$$

If $E$ were zero, then $D$ would be positive, and hence the right-hand side of (92) would diverge as $\lambda \rightarrow \infty$, which contradicts the fact that the left-hand side of (92) is bounded in $\lambda>0$. Hence, we obtain $E>0$.

Taking the limit as $\lambda \rightarrow \infty$ in both sides of formula (92), we have

$$
\mathbf{P}_{0}^{h}\left(L_{\infty}^{x}=L_{\infty}^{y}=0\right)=\frac{D}{4 E}
$$

which is nothing else but the second equality of (90). By formula (85), we obtain

$$
\begin{aligned}
\mathbf{P}_{0}^{h}\left(L_{\infty}^{x}=0, L_{\infty}^{y}>0\right)= & \mathbf{P}_{0}^{h}\left(L_{\infty}^{x}=0\right) \\
& -\mathbf{P}_{0}^{h}\left(L_{\infty}^{x}=L_{\infty}^{y}=0\right)=\frac{1}{2}-\frac{D}{4 E} .
\end{aligned}
$$

Thus we obtain (91). Therefore, we obtain

$$
\mathbf{P}_{0}^{h}\left(L_{\infty}^{x}>0, L_{\infty}^{y}>0\right)=1-\frac{D}{4 E}-2\left\{\frac{1}{2}-\frac{D}{4 E}\right\}=\frac{D}{4 E},
$$

which is nothing else but the first equality of (90). The proof is now complete.

5.4. Joint Law of $L_{\infty}^{x}$ and $L_{\infty}^{y}$. Let us discuss the joint law of $L_{\infty}^{x}$ and $L_{\infty}^{y}$ for $x, y \in \mathbb{R} \backslash\{0\}$ such that $x \neq y$.

By Lemma 11, we know that $D \geq 0$. First, we discuss the case of $D=0$.
Theorem 15. Suppose that $h(x)+h(y)-h(x-y)=0$. Then

$$
\begin{aligned}
& \mathbf{P}_{0}^{h}\left(h(x) L_{\infty}^{x} \in d l_{1}, h(y) L_{\infty}^{y} \in d l_{2}\right) \\
& \quad=\frac{1}{2}\left\{e^{-l_{1} / 2} \frac{d l_{1}}{2} \cdot \delta_{0}\left(d l_{2}\right)+\delta_{0}\left(d l_{1}\right) \cdot e^{-l_{2} / 2} \frac{d l_{2}}{2}\right\} .
\end{aligned}
$$

Proof. Since $D=0$ and $E=1$, formula (80) implies

$$
\begin{gathered}
\mathbf{P}_{0}^{h}\left[\exp \left\{-\lambda_{1} h(x) L_{\infty}^{x}-\lambda_{2} h(y) L_{\infty}^{y}\right\}\right] \\
=\frac{1+\lambda_{1}+\lambda_{2}}{1+2 \lambda_{1}+2 \lambda_{2}+4 \lambda_{1} \lambda_{2}}
\end{gathered}
$$

We may rewrite the right-hand side as

$$
\frac{1}{2}\left(\frac{1}{1+2 \lambda_{1}}+\frac{1}{1+2 \lambda_{2}}\right)
$$

which proves the claim.

Second, we discuss the case of $D>0$.

Theorem 16. Suppose that $h(x)+h(y)-h(x-y)>0$. Set

$$
\begin{gathered}
m=m(x, y)=h(x)+h(y)-h(x-y), \\
M=M(x, y)=\frac{4 h(x) h(y)}{h(x)+h(y)-h(x-y)} .
\end{gathered}
$$

Then one has $E>0$ and $0<m<M$. For any $A, B \in$ $\mathscr{B}([0, \infty))$, one has

$$
\mathbf{P}_{0}^{h}\left(h(x) L_{\infty}^{x} \in A, h(y) L_{\infty}^{y} \in B\right)=\sum_{k=1}^{4} C_{k} \Phi_{k}(A \times B),
$$

where $C_{k}=C_{k}(x, y), k=1,2,3,4$ are constants given as

$$
\begin{gathered}
C_{1}=\frac{D}{4 E}, \quad C_{3}=C_{4}=\frac{1}{2 E}\left(1-\frac{D}{2 E}\right), \\
C_{2}=1-C_{1}-C_{3}-C_{4}
\end{gathered}
$$

and $\Phi_{k}, k=1,2,3,4$ are positive measures on $[0, \infty)^{2}$ such that

$$
\begin{gathered}
\Phi_{1}(A \times B)=\delta_{0}(A) \delta_{0}(B), \\
\Phi_{2}(A \times B)=\mathbf{Q}_{0}^{2}\left(\frac{X_{m}}{m} \in A, \frac{X_{M}}{M} \in B\right) \\
=\mathbf{Q}_{0}^{2}\left(\frac{X_{M}}{M} \in A, \frac{X_{m}}{m} \in B\right), \\
\times B)=\Phi_{4}(B \times A) \\
=\mathbf{Q}_{0}^{2}\left[\frac{X_{m}}{m} \in A ; \mathbf{Q}_{X_{m}}^{0}\left(\frac{X_{M-m}}{M} \in B\right)\right] .
\end{gathered}
$$$$
\Phi_{3}(A \times B)=\Phi_{4}(B \times A)
$$

Remark 17. The expression (104) coincides with

$$
\mathbf{n}^{(0)}\left[2 m X_{m} ; \frac{X_{m}}{m} \in A, \frac{X_{M}}{M} \in B\right],
$$

where $\mathbf{n}^{(0)}$ is the generalized excursion measure introduced in Section 3. 
The proof of Theorem 16 will be given in the next section.

Remark 18. In the case where $\alpha=2$, the process $\left(\left(X_{t} / \sqrt{2}\right), \mathbf{P}_{0}^{h}\right)$ is the symmetrized three-dimensional Bessel process. In other words, if we set

$$
\begin{aligned}
& \Omega_{+}=\{w \in \mathbb{D}: \forall t>0, w(t)>0\}, \\
& \Omega_{-}=\{w \in \mathbb{D}: \forall t>0, w(t)<0\},
\end{aligned}
$$

then we have

$$
\mathbf{P}_{0}^{h}\left(\Omega_{+}\right)=\mathbf{P}_{0}^{h}\left(\Omega_{-}\right)=\frac{1}{2}
$$

and the processes $\left(\left(X_{t} / \sqrt{2}\right), \mathbf{P}_{0}^{h}\left(\cdot \mid \Omega_{+}\right)\right)$and $\left(\left(-X_{t} / \sqrt{2}\right)\right.$, $\left.\mathbf{P}_{0}^{h}\left(\cdot \mid \Omega_{-}\right)\right)$are one-sided three-dimensional Bessel processes. Hence, the Ray-Knight theorem implies that the process $\left(x^{2} L_{\infty}^{x}: x \geq 0\right)$ conditional on $\Omega_{+}$is the squared Bessel process of dimension two. Let us check that Theorems 15 and 16 are consistent with this fact. Since $h(x)=|x| / 2$, we have

$$
\begin{aligned}
h(x)+h(y)-h(x-y) & =\frac{|x|+|y|-|x-y|}{2} \\
& = \begin{cases}\min \{|x|,|y|\} & \text { if } x y>0, \\
0 & \text { if } x y<0 .\end{cases}
\end{aligned}
$$

If $x>0>y$, then we should look at Theorem 15 which implies that

$$
\mathbf{P}_{0}^{h}\left(L_{\infty}^{x} \in A, L_{\infty}^{y} \in B \mid \Omega_{+}\right)=\mathbf{Q}_{0}^{2}\left(X_{1} \in A\right) \cdot \delta_{0}(B) .
$$

If $x, y>0$, then we should look at Theorem 16 . Note that

$$
\begin{aligned}
& m(x, y)=\min \{x, y\}, \quad M(x, y)=\max \{x, y\} \\
& D=\frac{2|x-y|}{\max \{x, y\}}, \quad E=\frac{|x-y|}{\max \{x, y\}}, \quad \frac{D}{4 E}=\frac{1}{2}
\end{aligned}
$$

and that

$$
C_{1}=C_{2}=\frac{1}{2}, \quad C_{3}=C_{4}=0
$$

Hence, Theorem 16 implies that

$$
\mathbf{P}_{0}^{h}\left(x^{2} L_{\infty}^{x} \in A, y^{2} L_{\infty}^{y} \in B \mid \Omega_{+}\right)=\mathbf{Q}_{0}^{2}\left(X_{x} \in A, X_{y} \in B\right) .
$$

5.5. Proof of Theorem 16. We give the proof of Theorem 16 . We divide the proofs into several steps.

Step 1. Since

$$
0<D \leq 2 E=2\left(1-\frac{m}{M}\right)
$$

we have $0<m<M$.
Step 2. Let us compute the Laplace transform:

$$
\mathbf{Q}_{0}^{2}\left[\exp \left\{-\lambda_{1} \frac{X_{m}}{m}-\lambda_{2} \frac{X_{M}}{M}\right\}\right] .
$$

By the Markov property, the right-hand side is equal to

$$
\mathbf{Q}_{0}^{2}\left[\exp \left\{-\lambda_{1} \frac{X_{m}}{m}\right\} \mathbf{Q}_{X_{m}}^{2}\left[\exp \left\{-\lambda_{2} \frac{X_{M-m}}{M}\right\}\right]\right] .
$$

By formula (39), this expectation is equal to

$$
\begin{aligned}
& \frac{1}{1+2\left(\lambda_{2} / M\right)(M-m)} \\
& \quad \times \mathbf{Q}_{0}^{2}\left[\exp \left\{-\left(\frac{\lambda_{1}}{m}+\frac{\lambda_{2} / M}{1+2\left(\lambda_{2} / M\right)(M-m)}\right) X_{m}\right\}\right] .
\end{aligned}
$$

Again by formula (39), this expectation is equal to

$$
\begin{aligned}
& \frac{1}{1+2\left(\lambda_{2} / M\right)(M-m)} \\
& \quad \frac{1}{1+2\left(\lambda_{1} / m+\left(\lambda_{2} / M\right) /\left(1+2\left(\lambda_{2} / M\right)(M-m)\right)\right) m} .
\end{aligned}
$$

Simplifying this quantity with $E=1-m / M$, we see that

$$
\iint e^{-\lambda_{1} l_{1}-\lambda_{2} l_{2}} \Phi_{2}\left(d l_{1} \times d l_{2}\right)=\frac{1}{1+2 \lambda_{1}+2 \lambda_{2}+4 E \lambda_{1} \lambda_{2}} .
$$

Note that this expression is invariant under interchange between $\lambda_{1}$ and $\lambda_{2}$, which proves the second equality of (103).

Step 3. Let us compute the Laplace transform:

$$
\mathbf{Q}_{0}^{2}\left[\exp \left\{-\lambda_{1} \frac{X_{m}}{m}\right\} \mathbf{Q}_{X_{m}}^{0}\left[\exp \left\{-\lambda_{2} \frac{X_{M-m}}{M}\right\}\right]\right] .
$$

By formula (39), this expectation is equal to

$$
\mathbf{Q}_{0}^{2}\left[\exp \left\{-\left(\frac{\lambda_{1}}{m}+\frac{\lambda_{2} / M}{1+2\left(\lambda_{2} / M\right)(M-m)}\right) X_{m}\right\}\right] .
$$

Using the equality between (116) and (118), we see that

$$
\iint e^{-\lambda_{1} l_{1}-\lambda_{2} l_{2}} \Phi_{3}\left(d l_{1} \times d l_{2}\right)=\frac{1+2 E \lambda_{2}}{1+2 \lambda_{1}+2 \lambda_{2}+4 E \lambda_{1} \lambda_{2}} .
$$

Now we also obtain

$$
\iint e^{-\lambda_{1} l_{1}-\lambda_{2} l_{2}} \Phi_{4}\left(d l_{1} \times d l_{2}\right)=\frac{1+2 E \lambda_{1}}{1+2 \lambda_{1}+2 \lambda_{2}+4 E \lambda_{1} \lambda_{2}} .
$$

Step 4. Noting that

$$
\iint e^{-\lambda_{1} l_{1}-\lambda_{2} l_{2}} \Phi_{1}\left(d l_{1} \times d l_{2}\right)=1,
$$


we sum up formulae (123), (118), (121), and (122), and we obtain

$$
\begin{gathered}
\sum_{k=1}^{4} C_{k} \iint e^{-\lambda_{1} l_{1}-\lambda_{2} l_{2}} \Phi_{k}\left(d l_{1} \times d l_{2}\right) \\
=\frac{1+\lambda_{1}+\lambda_{2}+D \lambda_{1} \lambda_{2}}{1+2 \lambda_{1}+2 \lambda_{2}+4 E \lambda_{1} \lambda_{2}} .
\end{gathered}
$$

By Lemma 11, we see that the right-hand side coincides with the Laplace transform of the joint law of $\left(L_{\infty}^{x}, L_{\infty}^{y}\right)$ under $\mathbf{P}_{0}^{h}$. By the uniqueness of Laplace transforms, we obtain the desired conclusion.

\section{The Laws of Total Local Times for Bridges}

In this section, we study the total local time of Lévy bridges and $h$-bridges.

6.1. The Laws of the Total Local Times for Lévy Bridges. Let us work with the Lévy bridge $\mathbf{P}_{0,0}^{t}$ and its local time $\left(L_{s}^{z}: 0 \leq s \leq\right.$ t) such that

$$
\int_{0}^{s} f\left(X_{u}\right) d u=\int f(z) L_{s}^{z} d z, \quad 0 \leq s \leq t, f \in \mathscr{B}_{+}(\mathbb{R})
$$

with $\mathbf{P}_{0,0}^{t}$-probability one. Let us study the law of the total local time $L_{t}^{z}$ under $\mathbf{P}_{0,0}^{t}$.

Theorem 19. For $t>0$, it holds that

$$
\mathbf{P}_{0,0}^{t}\left(L_{t}^{0} \in d l\right)=\frac{\gamma_{l}(t)}{p_{t}(0)} d l
$$

Proof. Using Theorem 7 with $n=1$ and $z_{1}=0$, we have

$$
\int_{0}^{\infty} e^{-q t} p_{t}(0) \mathbf{P}_{0,0}^{t}\left[e^{-\lambda L_{t}^{0}}\right] d t=\frac{1}{1 / u_{q}(0)+\lambda} .
$$

By formula (61), we have

$$
\begin{aligned}
\frac{1}{1 / u_{q}(0)+\lambda} & =\int_{0}^{\infty} e^{-\left(1 / u_{q}(0)-\lambda\right) l} d l \\
& =\int_{0}^{\infty} \mathbf{P}_{0}\left[e^{-q \tau(l)}\right] e^{-\lambda l} d l .
\end{aligned}
$$

Hence, using Lemma 9, we obtain (126) by the Laplace inversion. The proof is now complete.

Theorem 20. For any $z \in \mathbb{R} \backslash\{0\}$, one has

$$
\begin{gathered}
\mathbf{P}_{0,0}^{t}\left(L_{t}^{z}=0\right)=\frac{p_{t}(0)-\left(\rho_{z} * \rho_{z} * p_{.}(0)\right)(t)}{p_{t}(0)}, \\
\mathbf{P}_{0,0}^{t}\left(L_{t}^{z} \in d l\right)=\frac{\left(\rho_{z} * \rho_{z} * \gamma_{l}\right)(t)}{p_{t}(0)} d l, \quad \text { for } l \neq 0,
\end{gathered}
$$

where the symbol $*$ stands for the convolution operation.
Proof. Using Theorem 7 with $n=2, \lambda_{1}=0, \lambda_{2}=\lambda, z_{1}=0$, and $z_{2}=z$, we have

$$
\begin{aligned}
\int_{0}^{\infty} e^{-q t} p_{t}(0) \mathbf{P}_{0,0}^{t}\left[e^{-\lambda L_{t}^{z}}\right] d t \\
\quad=u_{q}(0)\left(1-\frac{u_{q}(z)^{2}}{u_{q}(0)^{2}}\right)+\frac{u_{q}(z)^{2}}{u_{q}(0)^{2}} \frac{1}{1 / u_{q}(0)+\lambda} .
\end{aligned}
$$

On the one hand, it follows from (57) that

$$
\begin{aligned}
u_{q}(0) & \left(1-\frac{u_{q}(z)^{2}}{u_{q}(0)^{2}}\right) \\
= & \left(\int_{0}^{\infty} e^{-q t} p_{t}(0) d t\right)\left(1-\mathbf{P}_{z}\left[e^{-q T_{\{0\}}}\right]^{2}\right) .
\end{aligned}
$$

This implies (129). On the other hand, by (61) and (57), we have

$$
\frac{u_{q}(z)^{2}}{u_{q}(0)^{2}} \frac{1}{1 / u_{q}(0)+\lambda}=\mathbf{P}_{z}\left[e^{-q T_{\{0\}}}\right]^{2} \int_{0}^{\infty} \mathbf{P}_{0}\left(e^{-q \tau(l)}\right) e^{-\lambda l} d l
$$

This implies (130). The proof is now complete.

6.2. The Laws of the Total Local Times for h-Bridges. Let us work with the $h$-bridge $\mathbf{P}_{0,0}^{h, t}$ and its local time $\left(L_{t}^{z}\right)$ such that

$$
\int_{0}^{s} f\left(X_{u}\right) d u=\int f(z) L_{s}^{z} h(z)^{2} d z, \quad 0 \leq s \leq t, \quad f \in \mathscr{B}_{+}(\mathbb{R})
$$

with $\mathbf{P}_{0,0}^{h, t}$-probability one. We give the Laplace transform formula for the law of the total local time $L_{t}^{z}$ under $\mathbf{P}_{0,0}^{h, t}$.

Lemma 21. For $z \in \mathbb{R} \backslash\{0\}$ and $\lambda \geq 0$, one has

$$
\begin{gathered}
\int_{0}^{\infty} e^{-q t} p_{t}^{h}(0,0) \mathbf{P}_{0,0}^{h, t}\left[1-e^{-\lambda h(z)^{2} L_{t}^{z}}\right] d t \\
=\frac{\left(u_{q}(z)^{2} / u_{q}(0)^{2}\right) \lambda}{1+u_{q}(0)\left\{1-u_{q}(z)^{2} / u_{q}(0)^{2}\right\} \lambda} .
\end{gathered}
$$

Proof. Using Theorem 8 with $n=1, \lambda_{1}=\lambda$, and $z_{1}=z$, we have

$$
\int_{0}^{\infty} e^{-q t} p_{t}^{h}(0,0) \mathbf{P}_{0,0}^{h, t}\left[1-e^{-\lambda L_{t}^{z}}\right] d t=\frac{u_{q}^{h}(0, z)^{2} \lambda}{1+u_{q}^{h}(z, z) \lambda} .
$$

By formulae (72), we obtain the desired formula.

\section{Concluding Remark}

We gave an explicit formula which describes the joint distribution of the total local times at two levels and we discussed several formulae related to the law of the total local times. However, we could not obtain any better result 
on the law of the total local time with space parameter. As we noted in Remark 3, a difficulty arises in the case of $h$ paths, which comes from the asymmetry of the matrix $\Sigma-\Sigma^{0}$. We also remark that we have no better result related to the law of total local time in the case where the Markov process is asymmetric. We left the further study of the law of the total local time for asymmetric Markov process with space parameter for future work.

\section{Acknowledgment}

K. Yano is partially supported by Inamori Foundation.

\section{References}

[1] D. Applebaum, Lévy Processes and Stochastic Calculus, vol. 116 of Cambridge Studies in Advanced Mathematics, Cambridge University Press, Cambridge, UK, 2nd edition, 2009.

[2] P. Imkeller and I. Pavlyukevich, "Lévy flights: transitions and meta-stability," Journal of Physics A, vol. 39, no. 15, pp. L237L246, 2006.

[3] D. Revuz and M. Yor, Continuous Martingales and Brownian Motion, Springer, Berlin, Germany, 3rd edition, 1999.

[4] M. Yor, Some Aspects of Brownian Motion. Part I, Lectures in Mathematics ETH Zürich, Birkhäuser, Basel, Switzerland, 1992.

[5] N. Eisenbaum and H. Kaspi, "A necessary and sufficient condition for the Markov property of the local time process," The Annals of Probability, vol. 21, no. 3, pp. 1591-1598, 1993.

[6] N. Eisenbaum, H. Kaspi, M. B. Marcus, J. Rosen, and Z. Shi, "A Ray-Knight theorem for symmetric Markov processes," The Annals of Probability, vol. 28, no. 4, pp. 1781-1796, 2000.

[7] K. Yano, "Excursions away from a regular point for onedimensional symmetric Lévy processes without Gaussian part," Potential Analysis, vol. 32, no. 4, pp. 305-341, 2010.

[8] K. Yano, Y. Yano, and M. Yor, "Penalising symmetric stable Lévy paths," Journal of the Mathematical Society of Japan, vol. 61, no. 3, pp. 757-798, 2009.

[9] K. Yano, Y. Yano, and M. Yor, "On the laws of first hitting times of points for one-dimensional symmetric stable Lévy processes," in Séminaire de Probabilités XLII, vol. 1979 of Lecture Notes in Mathematics, pp. 187-227, Springer, Berlin, Germany, 2009.

[10] K. Yano, "Two kinds of conditionings for stable Lévy processes," in Probabilistic Approach to Geometry, vol. 57 of Advanced Studies in Pure Mathematic, pp. 493-503, Mathematical Society of Japan, Tokyo, Japan, 2010.

[11] R. M. Blumenthal and R. K. Getoor, Markov Processes and Potential Theory, vol. 29 of Pure and Applied Mathematics, Academic Press, New York, NY, USA, 1968.

[12] J. Pitman, "Cyclically stationary Brownian local time processes," Probability Theory and Related Fields, vol. 106, no. 3, pp. 299329, 1996.

[13] J. Pitman, "The distribution of local times of a Brownian bridge," in Séminaire de Probabilités, XXXIII, vol. 1709 of Lecture Notes in Mathematics, pp. 388-394, Springer, Berlin, Germany, 1999.

[14] J. Pitman and M. Yor, "A decomposition of Bessel bridges," Zeitschrift für Wahrscheinlichkeitstheorie und Verwandte Gebiete, vol. 59, no. 4, pp. 425-457, 1982.

[15] P. Fitzsimmons, J. Pitman, and M. Yor, "Markovian bridges: construction, Palm interpretation, and splicing," in Seminar on Stochastic Processes: Proceedings from 12th Seminar on Stochastic
Processes, 1992, University of Washington, Seattle, Wash, USA, 1992, vol. 33 of Progress in Probability Series, pp. 101-134, Birkhäauser, Boston, Mass, USA, 1993.

[16] M. B. Marcus and J. Rosen, Markov Processes, Gaussian Processes, and Local Times, vol. 100 of Cambridge Studies in Advanced Mathematics, Cambridge University Press, Cambridge, UK, 2006.

[17] J. Pitman and M. Yor, "Decomposition at the maximum for excursions and bridges of one-dimensional diffusions," in Itô's Stochastic Calculus and Probability Theory, pp. 293-310, Springer, Tokyo, Japan, 1996.

[18] P. J. Fitzsimmons and K. Yano, "Time change approach to generalized excursion measures, and its application to limit theorems," Journal of Theoretical Probability, vol. 21, no. 1, pp. 246-265, 2008.

[19] J. Bertoin, Lévy Processes, vol. 121 of Cambridge Tracts in Mathematics, Cambridge University Press, Cambridge, UK, 1996.

[20] S. Watanabe, K. Yano, and Y. Yano, "A density formula for the law of time spent on the positive side of one-dimensional diffusion processes," Journal of Mathematics of Kyoto University, vol. 45, no. 4, pp. 781-806, 2005. 


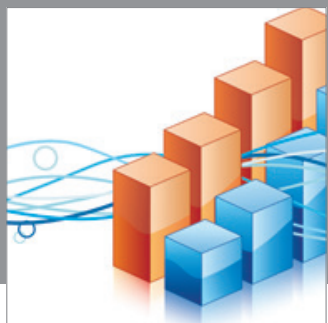

Advances in

Operations Research

mansans

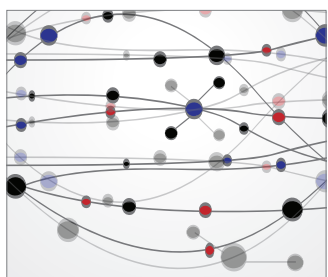

The Scientific World Journal
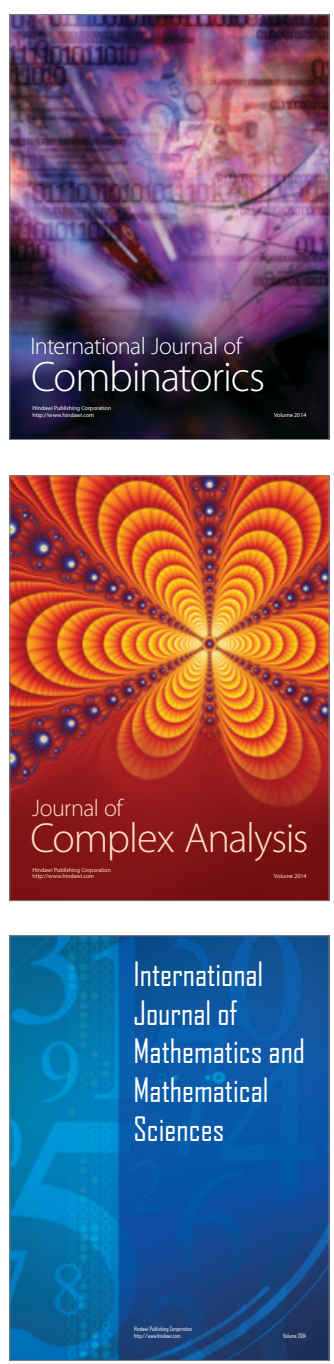
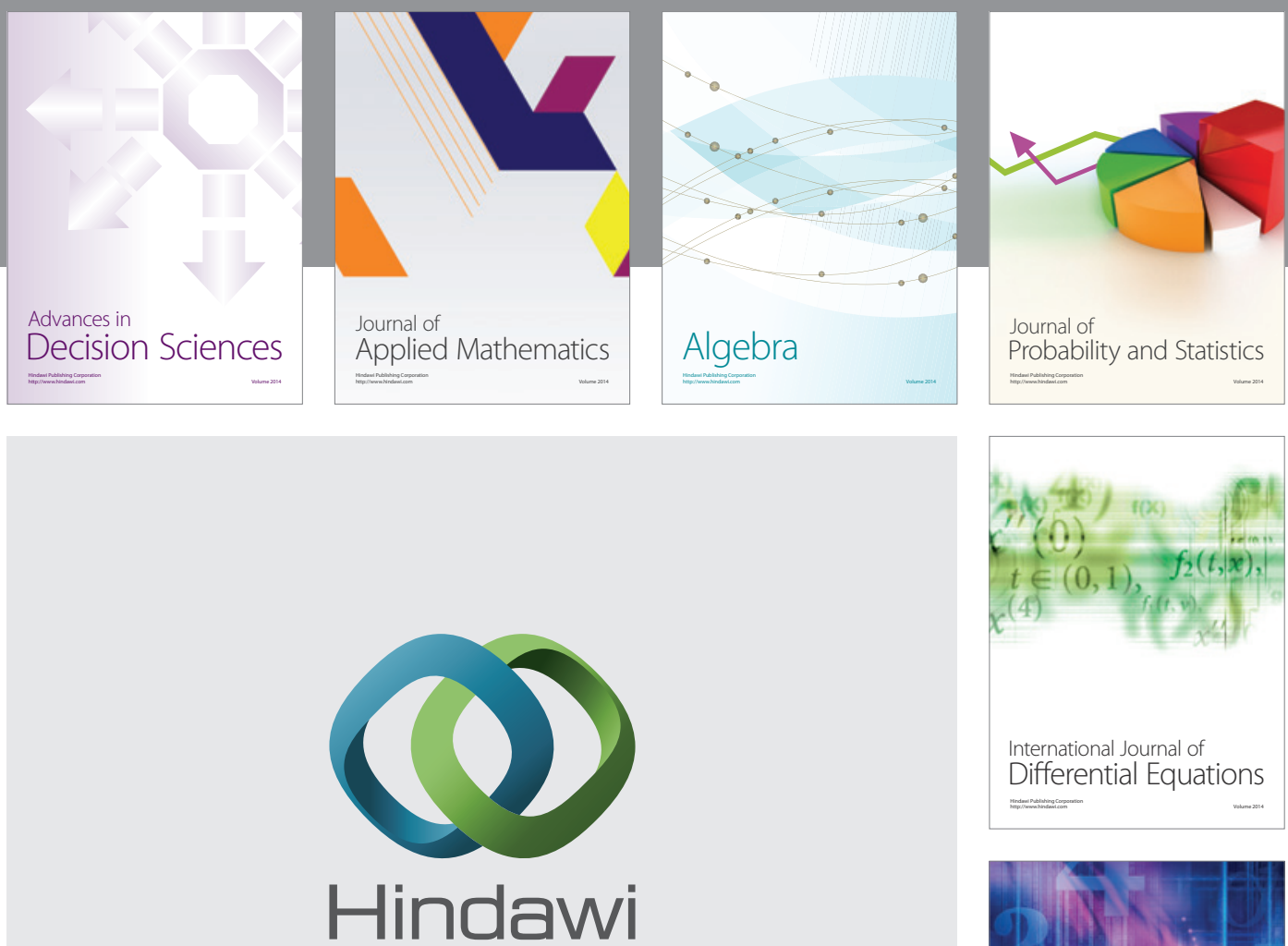

Submit your manuscripts at http://www.hindawi.com
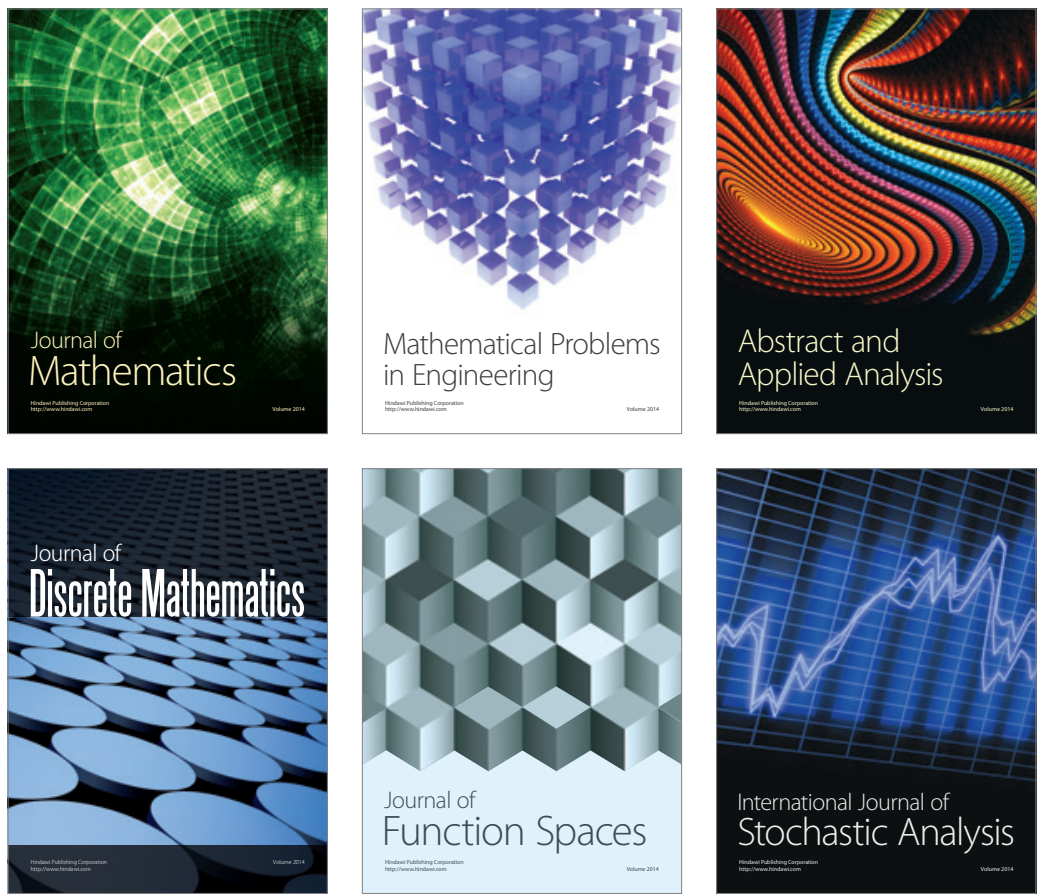

Journal of

Function Spaces

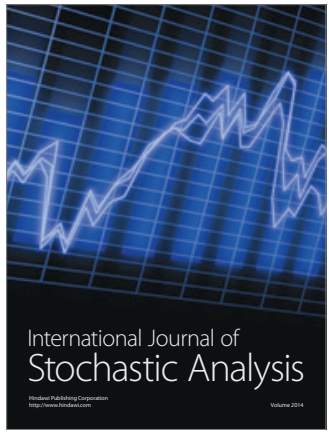

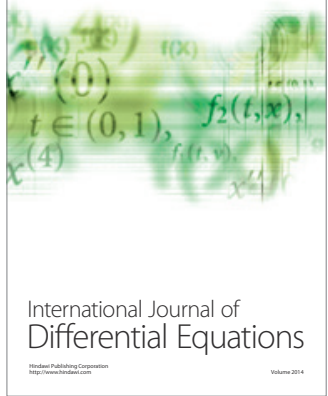
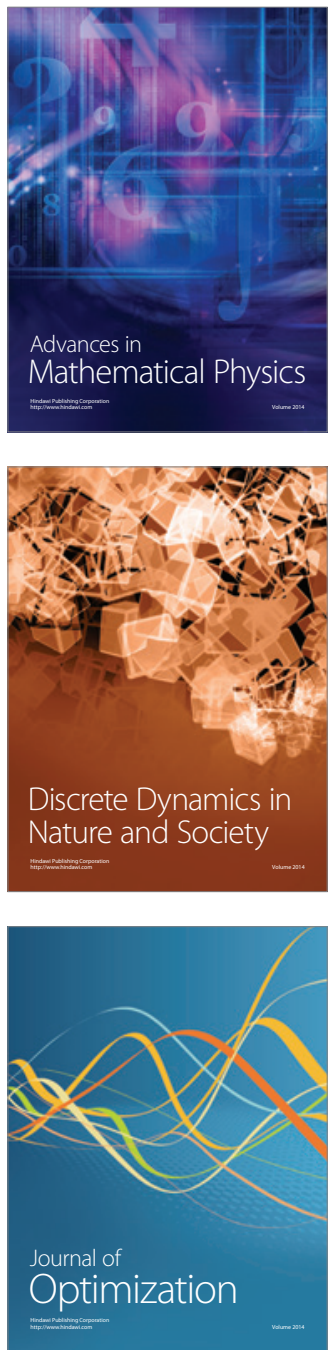